Please note, this is the uncorrected text of the proofs. Page numbers may not correspond to the printed version.

Philosophy and Phenomenological Research

Vol. LXX, No. 2, March 2005

\title{
Is Semantic Information Meaningful Data?
}

\author{
LUCIANO FLORIDI \\ Wolfson College
}

\begin{abstract}
There is no consensus yet on the definition of semantic information. This paper contributes to the current debate by criticising and revising the Standard Definition of semantic Information (SDI) as meaningful data, in favour of the Dretske-Grice approach: meaningful and well-formed data constitute semantic information only if they also qualify as contingently truthful. After a brief introduction, SDI is criticised for providing necessary but insufficient conditions for the definition of semantic information. SDI is incorrect because truth-values do not supervene on semantic information, and misinformation (that is, false semantic information) is not a type of semantic information, but pseudo-information, that is not semantic information at all. This is shown by arguing that none of the reasons for interpreting misinformation as a type of semantic information is convincing, whilst there are compelling reasons to treat it as pseudo-information. As a consequence, SDI is revised to include a necessary truth-condition. The last section summarises the main results of the paper and indicates some interesting areas of application of the revised definition.
\end{abstract}

\section{Introduction}

The concept of information has become central in most contemporary philosophy. However, recent surveys have shown no consensus on a single, unified definition of semantic information. ${ }^{1}$ This is hardly surprising. Information is such a powerful and elusive concept that, as an explicandum, it can be associated with several explanations, depending on the cluster of requirements and desiderata that orientate a theory. ${ }^{2}$ Claude Shannon, for example, remarked that

The word "information" has been given different meanings by various writers in the general field of information theory. It is likely that at least a number of these will prove sufficiently useful in certain applications to deserve further study and permanent recognition. It is hardly to be expected that a single concept of information would satisfactorily account for the numerous possible applications of this general field. From "The Lattice Theory of Information", in Shannon (1993) p. 180.

For a review of the literature and further information see Floridi [2002], [2003a], [2003b].

2 The point is made explicit and defended in Bar-Hillel and Carnap [1953], Szaniawski [1984] and Floridi [2002]. 
Polysemantic concepts such as information can be fruitfully analysed only in relation to well-specified contexts of application. Following this localist principle, only one crucial aspect of a specific type of information will be analysed in this paper, namely the alethic nature of declarative, objective and semantic (DOS) information (more on these qualifications in the next section). The question addressed is whether alethic values are supervenient ${ }^{3}$ on DOS information, as presumed by the standard definition of information (SDI). The negative answer defended is that DOS information encapsulates "truthfulness", so that "true information" is simply redundant and "false information", i.e. misinformation, is merely pseudo-information. It follows that SDI needs to be revised by adding a necessary truth-condition. Five areas of application of the revised definition are briefly discussed in the last section.

\section{The Standard Definition of Information}

Intuitively, "information" is often used to refer to non-mental, user-independent, declarative (i.e. alethically qualifiable) ${ }^{4}$ semantic contents, embedded in physical implementations like databases, encyclopaedias, web sites, television programmes and so on, which can variously be produced, collected, accessed and processed. The Cambridge Dictionary of Philosophy, for example, defines information thus:

an objective (mind independent) entity. It can be generated or carried by messages (words, sentences) or by other products of cognizers (interpreters). Information can be encoded and transmitted, but the information would exist independently of its encoding or transmission.

The extensionalist analysis of this popular concept of DOS (declarative, objective and semantic) information is not immediately connected to levels of subjective uncertainty and ignorance, to probability distributions, to utility-functions for decision-making processes, or to the analysis of communication processes. So the corresponding mathematical and pragmatic ${ }^{5}$ senses in

3 This technical term is used here to mean, weakly, "coming upon something subsequently, as an extraneous addition". The term is not used with the stronger meaning according to which "if a set of properties $x$ supervenes on another set of properties $y$, this means that there is no variation with respect to $\mathrm{x}$ without a variation with respect to $\mathrm{y}$ ". I am grateful to Philipp Keller for having prompted me to add this clarification.

4 There are many plausible contexts in which a stipulation ("let the value of $\mathrm{x}=3$ " or "suppose we discover the bones of a unicorn"), an invitation ("you are cordially invited to the college party"), an order ("close the window!"), an instruction ("to open the box turn the key"), a game move ("1.e2-e4 c7-c5" at the beginning of a chess game) may be correctly qualified as kinds of information. These and other similar, non-declarative meanings of "information" (e.g. to refer to a music file or to a digital painting) are not discussed in this paper, where objective semantic information is taken to have a declarative or factual value, i.e., it is suppose to be correctly qualifiable alethically.

5 See Bar-Hillel and Carnap [1953]. A pragmatic theory of information addresses the question of how much information a certain message carries for a subject $\mathrm{S}$ in a given doxastic state and within a specific informational environment. 
which one may speak of information are not relevant in this context and can be disregarded.

Over the last three decades, most analyses have supported a definition of DOS information in terms of data + meaning. Three quotations from a variety of influential texts well illustrate the popularity of the bipartite account: ${ }^{6}$

Information is data that has been processed into a form that is meaningful to the recipient. Davis and Olson (1985), 200.

Data is the raw material that is processed and refined to generate information. Silver and Silver (1989), 6.

Information equals data plus meaning. Checkland and Scholes (1990), 303.

The bipartite account has gained sufficient consensus to become an operational standard in fields that tend to deal with data and information as reified entities (consider, for example, the now common expression "data mining"), especially Information Science; Information Systems Theory, Methodology, Analysis and Design; Information (Systems) Management; Database Design; and Decision Theory. More recently, the bipartite account has begun to influence the philosophy of computing and information as well (see for example Chalmers [1996], Floridi [1999], Franklin [1995] and Mingers [1997]).

The practical utility of the bipartite account is indubitable. The question is whether it is rigorous enough to be applied in the context of an informationtheoretical epistemology. We shall see that this is not the case, but before moving any criticism, we need a more rigorous formulation.

\subsection{An Analysis of the Standard Definition of Information}

Situation logic (Israel and Perry [1990]; Devlin [1991]) provides a powerful methodology for our task. Let us use the symbol $\sigma$ and the term "infon" to refer to discrete items of information, irrespective of their semiotic code and physical implementation:

SDI) $\sigma$ is an instance of DOS information if and only if:

SDI.1) $\sigma$ consists of $n$ data (d), for $n \geq 1$;

SDI.2) the data are well-formed (wfd);

SDI.3) the wfd are meaningful $(\operatorname{mwfd}=\delta)$.

Three comments are now in order.

6 Many other sources endorse equivalent accounts as uncontroversial, see Floridi [2003a] for references. 
First, SDI.1 indicates that information cannot be dataless, but it does not specify which types of $\delta$ constitute information. Data can be of four types (Floridi [1999]):

$\delta .1)$ primary data. These are what we ordinarily mean by, and perceive as, the principal data stored in a database, e.g. a simple array of numbers, or the contents of books in a library. They are the data an information management system is generally designed to convey to the user in the first place;

ס.2) metadata. These are secondary indications about the nature of the primary data. They enable a database management system to fulfil its tasks by describing essential properties of the primary data, e.g. location, format, updating, availability, copyright restrictions, etc.;

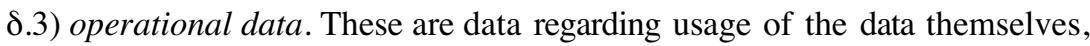
the operations of the whole data system and the system's performance;

$\delta .4)$ derivative data. These are data that can be extracted from $\delta .1-\delta .3$, whenever the latter are used as sources in search of patterns, clues or inferential evidence, e.g. for comparative and quantitative analyses (ideometry).

At first sight, the typological neutrality (TN) implicit in SDI.1 may seem counterintuitive. A database query that returns no answer, for example, still provides some information, if only negative information; and silence is a meaningful act of communication, if minimalist, Grice docet, yet where are the data in these cases? SD.1 and TN cannot be justified by arguing that absence of data is usually uninteresting, because similar pragmatic considerations are at least controversial, as shown by the previous two examples, and in any case irrelevant, since in this context the analysis concerns only DOS information, not interested information. ${ }^{7}$ Rather, SD.1 and TN are justified by the following principle of data-types reduction (PDTR):

PDTR) $\sigma$ consists of a non-empty set $(D)$ of data $\delta$; if $D$ seems empty and $\sigma$ still seems to qualify as information, then

1. the absence of $\delta$ is only apparent because of the occurrence of some negative primary $\delta$, so that $D$ is not really empty; or

2. the qualification of $\sigma$ as information consisting of an empty $D$ is misleading, since what really qualifies as information is not $\sigma$ itself but some

7 Interested information is a technical expression. The pragmatic theory of interested information is crucial in Decision Theory, where a standard quantitative axiom states that, in an ideal context and ceteris paribus, the more informative $\sigma$ is to $\mathrm{S}$, the more $\mathrm{S}$ ought to be rationally willing to pay to find out whether $\sigma$ is true [Sneed [1967]]. 
non-primary information $\mu$ concerning $\sigma$, constituted by meaningful nonprimary data $\delta .2-\delta .4$ about $\sigma$.

So in either case there is information because there is some type of data. Consider the two examples above. Suppose we are using the Routledge Encyclopedia of Philosophy on CD-ROM (EREP). If the database provides an answer, it will provide at least a negative answer, e.g. the EREP will open a small window with the message "no search hits found", so PDTR.1 applies: primary information is provided through explicit negative data. If the database provides no answer, either it fails to provide any data at all (e.g. the screen of the EREP remains unmodified), in which case no primary information $\sigma$ is available or, more likely, there is a way of monitoring or inferring the problems encountered by the database to establish, for example, that the EREP is not responding rather than being busy elaborating (one can try to use the CTRL + ALT + DEL command, which will open a Window with information about the performance of the programs currently open), in which case PDTR.2 applies: there isn't any primary information and the non-primary information gained is provided by some metadata. Take now the second example. My wife's silence could provide some primary information, e.g. a tacit assent or denial. The datum is the silence itself, as long as it counts as a difference (more on this in a moment). Or her silence could carry some nonprimary information $\mu$, e.g. she has not heard my question. The fact that I do not even know whether her silence provides some primary or non-primary information (let alone being able to guess the specific meaning of her silence) explains why, in any binary communication, we tend to adopt a "positive" signal for a negative message: the computer will send me a 1 or a 0 (primary negative information) or nothing at all (secondary negative information), rather than a signal or nothing at all, although the latter code would still be sufficient to communicate in ideal circumstances. This point can be further clarified by a third example. Imagine a very boring device that can produce only one symbol, like E. A. Poe's raven, who can answer only "nevermore." This is called a unary device. The raven is the informer, we are the informee, "nevermore" is the message, there is a coding and decoding procedure through a language, a channel of communication, and perhaps some possible noise. Informer and informee share the same background knowledge about the collection of usable symbols (the alphabet). Given this a priori knowledge, it is obvious that a unary device produces zero amount of primary information. Simplifying, we already know the outcome so our ignorance cannot be decreased. Whatever the informational state of the system, asking appropriate questions to the raven does not make any difference. The point that interests us here is that a unary source like the raven answers every question all the time with only one symbol, not with silence or symbol, since silence, if possible, would count as a signal, i.e. as a 0 . On the contrary, my wife's 
silence provides primary information if it is like a tacit 0 , that is, only if I assume that she might have answered something else instead. A completely silent source is equivalent to my wife not hearing the question and qualifies as a unary source, which can provide only non-primary information. This shows that although there is no dataless information, the presence of data, e.g. "nevermore", does not guarantee the presence of primary information.

To summarise, when apparent absence of $\delta$ is not reducible to the occurrence of negative primary $\delta$ (the equivalent of a zero), either there is no information or what becomes available and qualifies as information is some further non-primary information $\mu$ about $\sigma$, constituted by some non-primary $\delta .2-\delta .4$. Now, differences in the reduction both of the absence of positive primary $\delta$ to the presence of negative primary $\delta$ and of $\sigma$ to $\mu$ (when $D$ is truly empty) warrant that there can be more than one $\sigma$ that may (misleadingly) appear to qualify as information and be equivalent to an apparently empty $D$. Not all silences are the same. However, since SDI.1 defines information in terms of $\delta$, without any further restriction on the typological nature of the latter, it is sufficiently general to capture primary (positive or negative) $\delta .1$ and non-primary data $\delta .2-\delta .4$ as well, and hence the corresponding special classes of information just introduced. As far as SDI.1 is concerned, SDI is correct: there can be no dataless information.

Second comment. According to SDI.1, $\sigma$ can consist of only a single datum. Information is usually conveyed by large clusters or patterns of wellformed, codified data, often alphanumeric, which are heavily constrained syntactically and already very rich semantically. However, in its simplest form a datum can be reduced to just a lack of uniformity, that is, a difference between the presence and the absence of e.g. silence or of a signal:

Dd $) d=(x \neq y)$

The dependence of information on the occurrence of syntactically well-formed clusters, strings or patterns of data, and of data on the occurrence of physically implementable differences, explains why information can be decoupled from one type of physical support in favour of another. Interpretations of this support-independence can vary quite radically, however, because Dd leaves underdetermined not only the logical type to which the relata belong (see $\mathrm{TN}$ ), but also the classification of the relata (taxonomic neutrality) and the kind of support that the implementation of their inequality may require (ontological neutrality).

Consider the taxonomic neutrality (TaxN) first. A datum is usually classified as the entity exhibiting the anomaly, often because the latter is perceptually more conspicuous or less redundant than the background conditions. However, the relation of inequality is binary and symmetric. A white sheet of paper is not just the necessary background condition for the occurrence of a 
black dot as a datum, it is a constitutive part of the datum itself, together with the fundamental relation of inequality that couples it with the dot. Nothing is a datum per se for being a datum is an external property. So SDI endorses the following thesis:

$\operatorname{TaxN}$ ) a datum is a relational entity.

Understood as relational entities, data are definable as constraining affordances, exploitable by a system as input of adequate queries that correctly semanticise them to produce information as output. In short, semantic information can also be described erotetically as data + queries (Floridi [1999]).

Consider next the ontological neutrality $(\mathrm{ON})$. By rejecting the possibility of dataless information, GDI endorses the following modest thesis:

ON) no information without data representation.

$\mathrm{ON}$ is often interpreted materialistically, as advocating the impossibility of physically disembodied information, through the equation "representation = physical implementation", thus:

S.1) no information without physical implementation.

S.1 is an inevitable assumption when working on the physics of computation, since computer science must necessarily take into account the physical properties and limits of the carriers of information. ${ }^{8}$ It is also the ontological assumption behind the Physical Symbol System Hypothesis in AI and Cognitive Science (Newell and Simon [1976]). However, ON does not specify whether, ultimately, the occurrence of every discrete state necessarily requires a material implementation of the data representations. Arguably, environments in which all entities, properties and processes are ultimately noetic (e.g. Berkeley, Spinoza), or in which the material or extended universe has a noetic or non-extended matrix as its ontological foundation (e.g. Pythagoras, Plato, Leibniz, Fichte, Hegel), seem perfectly capable of upholding ON without embracing S.1. The relata in Dd could be monads, for example. Indeed, the classic realism vs. antirealism debate can be reconstructed precisely in terms of reasonably acceptable interpretations of ON.

All this explains why SDI is also consistent with two other popular slogans, this time favourable to the proto-physical nature of information and hence completely antithetic to S.1:

S.2) "It from bit. Otherwise put, every "it"- every particle, every field of force, even the space-time continuum itself-derives its function, its mean-

Landauer [1996]. The debate on S.1 has flourished especially in the context of quantum computing. 
ing, its very existence entirely - even if in some contexts indirectly-from the apparatus-elicited answers to yes-or-no questions, binary choices, bits. "It from bit" symbolizes the idea that every item of the physical world has at bottom - a very deep bottom, in most instances—an immaterial source and explanation; that which we call reality arises in the last analysis from the posing of yes-no questions and the registering of equipment-evoked responses; in short, that all things physical are information-theoretic in origin and that this is a participatory universe." Wheeler (1990), 5 .

and

S.3) "[information is] a name for the content of what is exchanged with the outer world as we adjust to it, and make our adjustment felt upon it." Wiener (1954), 17. "Information is information, not matter or energy. No materialism which does not admit this can survive at the present day" Wiener (1961), 132.

S.2 endorses an information-theoretic, metaphysical monism: the universe's essential nature is digital, being fundamentally composed of information as data instead of matter or energy, with material objects as a complex secondary manifestation. S.2 may, but does not have to endorse a computational view of information processes. S.3 advocates a more pluralistic approach along similar lines. Both are compatible with SDI.

The third and final comment concerns SDI.3 and can be introduced by discussing a fourth slogan:

S.4) "In fact, what we mean by information - the elementary unit of information-is a difference which makes a difference". Bateson (1973), 428.

S.4 is one of the earliest and most popular formulations of SDI (see for example Franklin [1995], 34 and Chalmers [1996], 281). A "difference" is just a discrete state, i.e. a datum, and "making a difference" simply means that the datum is "meaningful", at least potentially. How data can come to have an assigned meaning and function in a semiotic system in the first place is one of the hardest problems in semantics. Luckily, the semanticisation of data need not detain us here because SDI.3 only requires the $\delta$ to be provided with a semantics already. The point in question is not how but whether data constituting semantic information can be correctly described as being meaningful independently of an informee. The genetic neutrality (GN) supported by SDI states that:

GN) $\delta$ can have a semantics independently of any informee. 
Before the discovery of the Rosetta Stone, Egyptian hieroglyphics were already regarded as information, even if their semantics was beyond the comprehension of any interpreter. The discovery of an interface between Greek and Egyptian did not affect the hieroglyphics' embedded semantics but its accessibility. This is the weak, conditional-counterfactual sense in which SDI.3 can speak of meaningful data being embedded in an information-carrier informeeindependently. GN supports the possibility of information without an informed subject, to adapt Popper's phrase. Meaning is not (at least not only) in the mind of the user. GN is to be distinguished from the stronger, realist thesis, supported for example by Dretske (1981), according to which data could also have their own semantics independently of an intelligent producer/informer. This is also known as environmental information, and a typical example is supposed to be provided by the concentric rings visible in the wood of a cut tree trunk, which may be used to estimate the age of the plant.

To summarise, insofar as SDI provides necessary conditions for $\sigma$ to qualify as DOS information, it also endorses four types of neutrality: TN, TaxN, ON and GN. These features represent an obvious advantage, as they make SDI perfectly scalable to more complex cases, and hence reasonably flexible in terms of applicability. However, by specifying that SDI.1-SDI.3 are also sufficient conditions, SDI further endorses a fifth type of alethic neutrality (AN) which turns out to be problematic. Let us see why.

\section{Alethic neutrality}

According to SDI, alethic values are not embedded in, but supervene on semantic information:

AN) meaningful and well-formed data qualify as information, no matter whether they represent or convey a truth or a falsehood or have no alethic value at all.

\section{It follows that}

FI) false information (including contradictions), i.e. misinformation, is a genuine type of DOS information, not pseudo-information;

TA) tautologies qualify as information; and

TI) "it is true that $\sigma$ " where $\sigma$ is a variable that can be replaced by any instance of genuine DOS information, is not a redundant expression; for 
example, "it is true" in the conjunction " "the earth is round' qualifies as information and it is true" cannot be eliminated without semantic loss."

None of these consequences seems ultimately defensible, and their rejection forces a revision of AN and hence of SDI. For the sake of simplicity, in the rest of this article only the rejection of FI will be pursued, following two strategies. The first consist in showing that none of the main reasons that could be adduced for interpreting false information as a type of information is convincing. This strategy is pursued in section four. The second strategy consists in showing that there are compelling reasons to treat false and tautological information as pseudo-information. This is argued in section five. Regarding TA, this is commonly assumed to be false in the philosophical literature on semantic information (see Floridi 2003c), but it is also crucially connected to the interpretation of mathematical and analytic truths, so a satisfactory discussion of its negation cannot be pursued here but must be left to another paper. Further arguments against AN could also be formulated on the basis of the literature on deflationary theories of truth and hence a criticism of TI. These arguments are not going to be rehearsed here because the development of this strategy, which has interesting consequences for the deflationary theories themselves, deserves an independent analysis that lies beyond the scope of this paper. I shall return to the issue in the conclusion, but only to clarify what may be expected from this line of reasoning.

\section{Nine bad reasons to think that false information is a type of semantic information}

Linguistically, the expression "false information" is common and perfectly acceptable. What is meant by it is often less clear, though. The American legislation on food disparagement provides an enlightening example.

Food disparagement is legally defined in the US as the wilful or malicious dissemination to the public, in any manner, of false information that a perishable food product or commodity is not safe for human consumption. "False information" is then defined, rather vaguely, as

"information not based on reasonable and reliable scientific inquiry, facts, or data" (Ohio legislation, http://www.ohiocitizen.org/campaigns/pesticides/veglibel.html);

"information that is not based on verifiable fact or on reliable scientific data or evidence" (Vermont legislation, http://www.leg.state.vt.us/docs/2000/bills/intro/h-190.htm);

9 Note that the conjunction of FI and TI presupposes two theses that are usually uncontroversial: (i) that information is strictly connected with, and can be discussed in terms of alethic concepts; and (ii) that any theory of truth should treat alethic values or concepts symmetrically. 
"information which is not based on reliable, scientific facts and reliable scientific data which the disseminator knows or should have known to be false" (Arkansas legislation, http://www .arkleg.state.ar.us/ftproot/bills/1999/htm/hb1938.htm).

In each case, false information is defined in the same way in which one could define a rotten apple, i.e. as if it were a "bad" type of information, vitiated by some shortcoming. Why? Suppose that there are going to be exactly two guests for dinner tonight, one of whom is in fact vegetarian. This is our situation $\mathrm{S}$. Let the false information about $\mathrm{S}$ be $\mathrm{FI}=$ "(A) there will be exactly three guests for dinner tonight and (B) one of them is vegetarian". One may wish to argue that FI is not mere pseudo-information, but a certain type of information that happens to be false, for a number of reasons, yet even the most convincing ones are not convincing enough. Let us see why:

FI.1) FI can include genuine information.

Objection: this merely shows that FI is a compound in which only the true component B qualifies as information.

FI.2) FI can entail genuine information.

Objection: even if one correctly infers only some semantically relevant and true information TI from FI, e.g. that "there will be more than one guest", what now counts as information is the inferred true consequence TI, not FI. Besides, ex falso quod libet sequitur, so any contradiction would count as information.

FI.3) FI can still be genuinely informative, if only indirectly.

Objection: this is vague, but it can be reduced to the precise concept of nonprimary information $\mu$ discussed in section two. For example, FI may be coupled to some true, metainformation $\mathrm{M}$ that the source of FI is not fully reliable. What now counts as information is the true M, not the false FI.

\section{FI.4) FI can support decision-making processes.}

Objection: one could certainly cook enough food on the basis of FI but this is only accidental. The actual situation $\mathrm{S}$ may be represented by a wedding dinner for a hundred people. That is why FI fails to qualify as information. However, FI.4 clarifies that, if FI is embedded in a context in which there is enough genuine metainformation about its margins of error, then FI can be epistemically preferable to, because more useful than, both a false $\mathrm{FI}_{1}$, e.g. "there will be only one guest for dinner", and a true but too vacuous $\mathrm{FI}_{2}$, e.g. "there will be less than a thousand guests for dinner". What this shows is not (i) that false information is an alethically qualified type of genuine informa- 
tion, but that (ii) false information can still be pragmatically interesting (in the technical sense of the expression, see section two), because sources of information are usually supposed to be truth-oriented or truth-tracking by default (i.e. if they are mistaken, they are initially supposed to be so only accidentally and minimally), and that (iii) logically, an analysis of the information content of $\sigma$ must take into account the level of approximation of $\sigma$ to its reference, both when $\sigma$ is true and when it is false.

FI.5) FI is meaningful and has the same logical structure as genuine information.

Objection: this is simply misleading. Consider the following FI: "One day we shall discover the biggest of all natural numbers". Being necessarily false, this can hardly qualify as genuine but false information. It can only provide some genuine, non-primary information $\mu$, e.g. about the mathematical naivety of the source. In the same sense in which hieroglyphics could qualify as information even when they were not yet interpretable, vice versa, an infon $\sigma$ does not qualify as information just because it is interpretable. This point is further discussed in section five.

FI.6) FI could have been genuine information had the relevant situation been different. Perhaps the difficulty seen in FI.5 is caused by the necessary falsehood of the example discussed. Meaningful and well-formed data that are only contingently false represent a different case and could still qualify as a type of information. It only happens that there will be fewer guests than predicted by FI.

Objection: this only shows that we are ready to treat FI as quasi-information in a hypothetical-counterfactual sense, which is just to say that, if S had been different then FI would have been true and hence it would have qualified as information. Since S is not, FI does not. FI need not necessarily be pseudoinformation. It may be so contingently. This point too is further discussed in section five.

FI.7) If FI does not count as information, what is it? Assuming that $p$ is false "if $\mathrm{S}$ only thinks he or she has information that $p$, then what does $\mathrm{S}$ really have? Another cognitive category beyond information or knowledge would be necessary to answer this question. But another cognitive category is not required because we already have language that covers the situation: $\mathrm{S}$ only thinks he or she has knowledge that $p$, and actually has only information that p." (Colburn [2000], 468).

Objection: first, a new cognitive category could be invented, if required; secondly, there is actually another cognitive category, that of well-formed and 
meaningful data, which, when false, constitute misinformation, not a type of information. Third, the difference between being informed that $p$ and knowing that $p$ is that, in the latter case, $\mathrm{S}$ is supposed to be able to provide, among other things, a reasonable and appropriate (possibly non-Gettierisable) account of why $p$ is the case. The student $\mathrm{Q}$ who can recall and state a mathematical theorem $p$ but has no understanding of $p$ or can provide no further justification for $p$, can be said to be at most informed that $p$ without having knowledge that $p$. But if the mathematical theorem is not correct (if $p$ is false), it must be concluded that $\mathrm{Q}$ is misinformed (i.e. not informed) that $p$ ( $\mathrm{Q}$ does not have any information about the theorem). It is perfectly possible, but strikes one as imprecise and conceptually unsatisfactory, to reply that $\mathrm{Q}$ is informed that $p$ (Q does have some information about the theorem) and $p$ is false.

FI.8) We constantly speak of FI. Rejecting FI as information means denying the obvious fact that there is plenty of information in the world that is not true.

Objection: insofar as DOC information is concerned, this is a non sequitur. Denying that FI counts as a kind of information is not equivalent to denying that FI is a common phenomenon; it is equivalent to denying that a false policeman, who can perfectly well exist, counts as a kind of policeman at all. We shall see this better in the next section. Here it is sufficient to acknowledge that ordinary uses of technical words may be too generic and idiosyncratic, if not incorrect, to provide conceptual guidelines.

FI.9) “ ' $\mathrm{x}$ misinforms $\mathrm{y}$ that $p$ ' entails that $\neg p$ but ' $\mathrm{x}$ informs $\mathrm{y}$ that $p$ ' does not entail that $p$ [and since] ... we may be expected to be justified in extending many of our conclusions about 'inform' to conclusions about 'information' [it follows that]... informing does not require truth, and information need not be true; but misinforming requires falsehood, and misinformation must be false." (Fox [1983], 160-1, 189, 193).

Objection: the principle of "exportation" (from information as process to information as content) is more than questionable, but suppose it is accepted; misinforming becomes now a way of informing and misinformation a type of information. All this is as odd as considering lying a way of telling the truth about something else and a contingent falsehood a type of truth on a different topic. The interpretation becomes perfectly justified, however, if informing/ information is used to mean, more generically, communicating/communication, since the latter does not entail any particular truth value. But then compare the difference between: (a) "Q is told that $p$ " and (b) "Q is informed that $p$ ", where in both cases $p$ is a contradiction. (a) does not have to entail that $p$ 
is true and hence it is perfectly acceptable, but (b) is more ambiguous. It can be read as meaning just "Q was made to believe that $p$ ", for example, in which case information is treated as synonymous with (a form of) communication (this includes teaching, indoctrination, brain-washing etc.), as presumed by FI.9. But more likely, one would rephrase it and say that (b) means "Q is misinformed that $p$ " precisely because $p$ is necessarily false, thus implying that it makes little sense to interpret (b) as meaning " $\mathrm{S}$ has the information that $p$ " because a contradiction can hardly qualify as information (more on this in section five) and being informed, strictly speaking, entails truth.

In conclusion, there seem to be no good reason to treat false information as a type of information. This negative line of reasoning, however, may still be unconvincing. We need more "constructive" arguments showing that false information is pseudo-information. This is the task of the next section.

\section{Two good reasons to believe that false information is pseudo-information}

The first positive argument is a test based on a conceptual clarification. The clarification is this. The confusion about the nature of false information seems to be generated by a misleading analogy. The most typical cases of misinformation are false propositions and incorrect data. Now a false proposition is still a proposition, even if it is further qualified as not being true. The same holds true for incorrect data. Likewise, one may think that misinformation is still a type of information, although it happens not to be true. The logical confusion here is between attributive and predicative uses of "false". The distinction was already known to medieval logicians, was revived by Geach (1956) and requires a further refinement before being applied as a test to argue that "false information" is pseudo-information.

Take two adjectives like "male" and "good". A male constable is a person who is both male and employed as a policeman. A good constable, however, is not a good person who is also employed as a member of the police force, but rather a person who performs all the duties of a constable well. "Male" is being used as a predicative adjective, whereas "good" modifies "constable" and is being used as an attributive adjective. On this distinction one can build the following test: if an adjective in a compound is attributive, the latter cannot be split up. This property of indivisibility means that we cannot safely predicate of an attributively-modified $\mathrm{x}$ what we predicate of an $\mathrm{x}$. So far Geach. We now need to introduce two further refinements. Pace Geach, at least some adjectives can be used attributively or predicatively depending on the context, rather than necessarily being classified as either attributive or predicative intrinsically. Secondly, the attributive use can be either positive or negative. Positive attributively-used adjectives further qualify their refer- 
ence x as y. "Good constable" is a clear example. Negative, attributively-used adjectives negate one or more of the qualities necessary for $\mathrm{x}$ to be $\mathrm{x}$. They can be treated as logically equivalent to "not". For example, a false constable (attributive use) is clearly not a specific type of constable, but not a constable at all (negative use), although the person pretending to be a constable may successfully perform all the duties of a genuine constable (this further explains FI.4 above). The same holds true for other examples such as "forged banknote", "counterfeit signature", "false alarm" and so on. They are all instances of a correct answer "no, it is a $\mathrm{F}(\mathrm{x})$ " to the type-question "is this a genuine $\mathrm{x}$ ?".

Let us now return to the problem raised by the analogy between a false proposition and false information. When we say that $p$, e.g. "the earth has two moons", is a false proposition, we are using "false" predicatively. The test is that the compound can be split into " $p$ is a proposition" and " $p$ is a contingent falsehood" without any semantic loss or confusion. On the contrary, when we describe $p$ as false information, we are using "false" attributively, to negate the fact that $p$ qualifies as information at all. Why? Because "false information" does not pass the test. As in the case of the false constable, the compound cannot be correctly split: it is not the case, and hence it would be a mistake or an act of misinformation to assert, that $p$ constitutes information about the number of natural satellites orbiting around the earth and is also a falsehood. Compare this case to the one in which we qualify $\sigma$ as digital information, which obviously splits into " $\sigma$ is information" and " $\sigma$ is digital". If false information were a genuine type of information it should pass the splitting test. It does not, so it is not.

The second argument is semantic and more technical but its gist can be outlined in rather simple terms. If false information does not count as semantic junk but as a type of information, it becomes difficult to make sense of the ordinary phenomenon of semantic erosion. Operators like "not" lose their semantic power to corrupt information, information becomes semantically indestructible and the informative content of a repository can decrease only by physical and syntactical manipulation of data. This is utterly implausible, even if not logically impossible. We know that the cloth of semantic information can be, and indeed is, often undone by equally semantic means. When false information is treated as semantic information, what may be under discussion is only a purely quantitative or syntactic concept of information, that is meaningful and well-formed data, not DOS information.

\section{The standard definition of information revised}

Well-formed and meaningful data may be of poor quality. Data that are incorrect (somehow vitiated by errors or inconsistencies), imprecise (understanding precision as a measure of the repeatability of the collected data) or inaccurate 
(accuracy refers to how close the average data value is to the "true" value) are still data and they are often recoverable, but, if they are not truthful, they can only constitute misinformation (let me repeat: they can be informative, but only indirectly or derivatively, for example they can be informative about the unreliability of a source, but this is not the issue here). We have seen that misinformation (false information) has turned out to be not a type of information but rather pseudo-information. This is the Dretske-Grice approach (other philosophers who accept a truth-based definition of semantic information are Barwise and Seligman [1997] and Graham [1999]):

[...] false information and mis-information are not kinds of information - any more than decoy ducks and rubber ducks are kinds of ducks. Dretske (1981), 45.

False information is not an inferior kind of information; it just is not information. Grice (1989), 371.

Like "truth" in the expression "theory of truth", "information" can be used as a synecdoche to refer both to "information" and to "misinformation". "False information" is like "false evidence": it is not an oxymoron, but a way of specifying that the contents in question do not conform to the situation they purport to map. This is why, strictly speaking, to exchange (receive, sell, buy, etc.) false DOS information about x, e.g. the number of moons orbiting around the earth, is to exchange (receive, sell, buy, etc.) no DOS information at all about $x$, only meaningful and well-formed data, that is semantic content. Since syntactical well-formedness and meaningfulness are necessary but insufficient conditions for information, it follows that SDI needs to be modified, to include a fourth condition about the positive alethic nature of the data in question:

RSDI) $\sigma$ is an instance of DOS information if and only if:

1. $\sigma$ consists of $n$ data (d), for $n \geq 1$;

2. the data are well-formed (wfd);

3. the wfd are meaningful $(\operatorname{mwfd}=\delta)$;

4. the $\delta$ are truthful.

"Truthful" is used here as synonymous for "true", to mean "representing or conveying true contents about the referred situation or topic". It is preferable to speak of "truthful data" rather than "true data" because the data in question may not be linguistic (a map, for example, is truthful rather than true) and because we have seen that "true data" may give rise to a confusion, as if one 
were stressing the genuine nature of the data in question, not their positive alethic value. ${ }^{10}$

\section{Conclusion: summary of results and future developments}

Whales are not fish just because one may conceptually think so or say so. Likewise, we ordinarily speak of false information when what we mean is misinformation, i.e. no information at all. The goal of this paper has been to clarify the confusion. The standard definition of DOS information (SDI) provides necessary but insufficient conditions for the qualification of data as information. The definition has been modified to take into account the fact that information encapsulates truthfulness. The new version of the definition (RSDI) now describes DOS information as well-formed, meaningful and truthful data. In the course of the analysis, the paper has provided an explanation and refinement of the three necessary conditions established by SDI; an analysis of the concept of data; a clarification of four popular interpretations of SDI; and a revision of some basic principles and requirements that underpin any theory of semantic information. It may be useful to list now at least five interesting areas of application of RSDI:

1) the critique of the deflationary theories of truth (DTT). From RSDI, it follows that one could accept deflationary arguments as perfectly correct while rejecting the explanatory adequacy of DTT. "It is true that" in "it is true that $\sigma$ " is redundant because there cannot be semantic information that is not true, but DTT could mistake this linguistic or conceptual redundancy for unqualified dispensability. "It is true that" is redundant precisely because, strictly speaking, information is not a truth-bearer but already encapsulates truth as truthfulness. Thus, DTT may be satisfactory as theories of truth-ascriptions while being inadequate as theories of truthfulness;

2) the analysis of the informative nature of mathematical and analytic propositions;

3) the debate on the informative nature of sentences containing "empty" names, such as "Sherlock Holmes is a bachelor" or "Unicorns do not exist" (a true negative existential); ${ }^{11}$

4) a revision of the analysis of the standard definition of knowledge as justified and true belief in light of a "continuum" hypothesis that knowledge encapsulates truth because it encapsulates DOS information;

10 I am grateful to Timothy Colburn and Philipp Keller for having pointed out this other possible source of confusion.

11 I am grateful to a PPR referee for having called my attention to the parallelism between the analysis of false information and the debate on the semantics of empty names. 
5) the development of a quantitative theory of semantic information based on truth-values and degrees of discrepancy of $\sigma$ with respect to a given situation rather than probability distributions (Bar-Hillel and Carnap [1953], Floridi [2003c]).

The development of these lines of research has been left to a second stage of this work. ${ }^{12}$

12 I wish to thank Frederick R. Adams, Ian C. Dengler, Roger Brownsword, Timothy Colburn, James Fetzer, Ken Herold, Bernard Katz, Philipp Keller, Janet D. Sisson, and J. L. Speranza for their valuable suggestions on previous drafts of this paper. A more polished version was used by Anthonie W. M. Meijers and his students in a series of lectures about the philosophical aspects of information at the Delft University of Technology, The Netherlands, and I am very grateful to him and to the students who attended the lectures for having shared with me their detailed comments. Finally, I am in debt to a PPR referee for his very useful comments on the last draft. Any remaining mistake is only mine. 


\section{References}

Bar-Hillel, Y. and Carnap, R. 1953, "An Outline of a Theory of Semantic Information", rep. in Y. Bar-Hillel, Language and Information (Reading, MA; London: Addison Wesley, 1964), pp. 221-274, page references are to this edition.

Barwise, J. and J. Seligman 1997, Information flow: the logic of distributed systems. (Cambridge: Cambridge University Press).

Bateson, G. 1973, Steps to an Ecology of Mind (Paladin. Frogmore, St. Albans).

Chalmers, D. J. 1997, The Conscious Mind: in Search of a Fundamental Theory (Oxford: Oxford University Press).

Checkland, P. B. and Scholes, J. 1990, Soft Systems Methodology in Action (New York: John Wiley \& Sons).

Colburn T. R. 2000, "Information, Thought, and Knowledge", Proceedings of the World Multiconference on Systemics, Cybernetics and Informatics (Orlando FL, July 23-26, 2000), vol. X, 467-471.

Davis, G. B. and Olson, M. H. 1985, Management Information Systems: Conceptual Foundations, Structure, and Development, $2^{\text {nd }}$ ed. (New York: McGraw-Hill).

Devlin, K. 1991, Logic and Information (Cambridge: Cambridge University Press).

Dretske, F. 1981, Knowledge and the Flow of Information (Cambridge, Ma: MIT Press, rep. Stanford: CSLI, 1999).

Floridi, L. 1999, Philosophy and Computing-An Introduction (London and New York: Routledge).

Floridi, L. 2002, "What is the Philosophy of Information?", Metaphilosophy 33.1-2, 123-145.

Floridi, L. 2003a (editor), The Blackwell Guide to the Philosophy of Computing and Information (Oxford and New York: Blackwell), see chapter five entitled "Information".

Floridi, L. 2003b, "Information, Semantic Conceptions of", forthcoming in the Stanford Encyclopedia of Philosophy.

Floridi, L. 2003c, "Outline of a Theory of Strongly Semantic Information", forthcoming in Minds and Machines.

Fox C. J. 1983, Information and Misinformation-An Investigation of the Notions of Information, Misinformation, Informing, and Misinforming (Westport, CT: Greenwood Press).

Franklin, S. 1995, Artificial Minds (Cambridge, MA: The MIT Press).

Geach, P. T. 1956, "Good and Evil”, Analysis 17, 33-42.

Graham, G. 1999, The Internet:// A Philosophical Inquiry (London and New York: Routledge). 
Grice, P. 1989, Studies in the Way of Words (Cambridge MA: Harvard University Press).

Israel, D. and Perry J. 1990, "What is Information?" in P. Hanson (ed.), Information, Language and Cognition (Vancouver: University of British Columbia Press), 1-19.

Landauer, R. 1996, “The Physical Nature of Information” Physics Letter (A 217), 188 .

Mingers, J. 1997, “The Nature of Information and Its Relationship to Meaning", in R. L. Winder, S. K. Probert and I. A. Beeson (eds.), Philosophical Aspects of Information Systems (London: Taylor and Francis, 1997), pp. 73-84.

Newell, A. and Simon, H. A. 1976, "Computer Science as Empirical Inquiry: Symbols and Search" Communications of the ACM, 19 (March), 113-126.

Shannon, C. E. 1993, Collected Papers ed. by N. J. A. Sloane and \& A. D. Wyner (Los Alamos, Ca: IEEE Computer Society Press).

Silver, G. A. and Silver, M. L. 1989, Systems Analysis and Design (Reading, MA: Addison Wesley).

Sneed, D. J. 1967, "Entropy, Information and Decision", Synthese 17, pp. 392-407.

Szaniawski, K. 1984, “On Defining Information”, now in K. Szaniawski, On Science, Inference, Information and Decision Making, Selected Essays in the Philosophy of Science, ed. by A. Chmielewski and J. Wolenski (Dordrecht: Kluwer, 1998).

Wheeler, J. A. 1990, "Information, Physics, Quantum: The Search for Links" in W. H. Zureck (ed.) Complexity, Entropy, and the Physics of Information (Redwood City, CA: Addison Wesley).

Wiener, N. 1954, The Human Use of Human Beings: Cybernetics and Society, $2^{\text {nd }}$ ed. (London), reissued in 1989 with a new introduction by Steve J. Heims (London: Free Association).

Wiener, N. 1961, Cybernetics or Control and Communication in the Animal and the Machine, $2^{\text {nd }}$ ed. (Cambridge, MA: MIT Press) 